\title{
Numerical Simulation and Analysis of a Saturated- Core Type Superconducting Fault Current Limiter
}

\author{
Yuelong Jia, Mark D. Ainslie, Member, IEEE, Di Hu, and Jiansheng Yuan
}

\begin{abstract}
The saturated-core type superconducting fault current limiter (SCSFCL) is an important device to limit increasing fault currents in electrical power grids. However, there have been few numerical simulations carried out to simulate the properties of the superconducting DC coil in the SCSFCL, but such modeling is indispensable to the design and analysis of such devices. In this paper, a simulation method to simulate the SCSFCL with COMSOL Multiphysics ${ }^{\circledR}$ is presented, and the AC losses of the DC coil in the normal, nonlimiting state, which is its usual operating state, are simulated and analyzed for the first time. A strategy to simulate the AC loss of the superconducting coil with a 3D model is given first. The non-linear electric and magnetic problems are decoupled first. Next, the model is divided into smaller models for separate calculations. The 3D model is further simplified into a $2 \mathrm{D}$ model based on the worst-case scenario for a conservative design. The AC loss distribution within the coil is given and the relationship between the AC loss and an AC component of the current is analyzed, and it is found that the outermost coil regions have much larger AC loss than the coils near the centre.
\end{abstract}

Index Terms - AC loss, finite-element analysis, hightemperature superconductors, numerical analysis, saturated core, superconducting coils, superconducting fault current limiter.

\section{INTRODUCTION}

ShORT-CIRCUIT fault currents continue to increase due to the $\mathcal{N}_{\text {growth in demand for electricity and increased complexity }}$ of electrical power systems. As the fault current approaches the maximum value that the circuit breaker is able to limit, the safety of the power grids is threatened [1, 2]. Traditional, widely-used measures to limit the fault current, like splitting bus bars and so on, may affect operation flexibility of power grids or contribute to operating losses [3, 4]. Therefore, effective and low energy-consuming devices to limit the shortcircuit fault current are required. With developments in high temperature superconducting (HTS) materials, the saturatedcore type superconducting fault current limiter (SCSFCL) has become a key device for limiting fault currents. There are two key features for such fault current limiters: in normal operation, they have a minimal effect on the power grid, and

Dr M. D. Ainslie is supported by a Royal Academy of Engineering Research Fellowship. Mr Y. Jia is supported by the National High-Tech R\&D Program of China (863 Program, No. 2014AA032705) and the China Scholarship Council.

Y. Jia and J. Yuan are with the State Key Laboratory of Control and Simulation of Power System and Generation Equipments, Tsinghua University, Beijing 100084, China. Y. Jia is also with the Bulk Superconductivity Group, University of Cambridge CB2 1PZ, U.K. (e-mail: rhinestone@163.com).

M. D. Ainslie and D. Hu are with the Bulk Superconductivity Group, Department of Engineering, University of Cambridge, Cambridge CB2 1PZ, U.K. (e-mail: mark.ainslie@eng.cam.ac.uk). when a short-circuit fault occurs, they can limit the shortcircuit fault current effectively.

Although much research has been carried out on SCSFCLs, this has usually focused mainly on the current limiting effect in the current-limiting state [5,6], the voltage-current characteristics of the AC copper coils in normal operation [7], or the energy efficiency in terms of the DC current multiplied by the number of turns [8]. There have been few numerical simulations carried out to simulate the properties of the superconducting DC coil in SCSFCL. However, there is induced current in the DC coil and a large leakage flux due to the saturation of the iron core, both of which will result in the decrease of the critical current of the DC coil and an increase in AC losses. Despite the fact that the AC loss may seem small, it is dissipated as heat in a low temperature environment, e.g., $77 \mathrm{~K}$, which will be amplified by the cooling system as a much larger loss in a room temperature environment. Therefore, simulating the properties of the superconducting DC coil in the normal, non-limiting state, which is its usual operating state, is indispensable to the design and analysis of such SCSFCLs.

It can be extremely difficult to carry out such calculations accurately. Firstly, there are two non-linear problems coupled with each other: the non-linear magnetic properties of the iron core, and the non-linear electrical properties of the superconductors. Two sets of non-linear equations significantly increase the complexity of the numerical model and its solution time. Secondly, these two non-linear materials have a large geometric scale difference, up to several million times. Finally, the superconducting DC coil itself consists of up to several thousand turns, and the superconducting wire comprising the coil has a large aspect ratio that requires a very fine mesh, which also increases the required computational time. This paper presents a simplified method with COMSOL Multiphysics ${ }^{\circledR}$, making the simulation of the superconducting DC coil in SCSFCL possible.

The working principles of the SCSFCL are presented first. Next, the simulation method to estimate the AC losses of the superconducting DC coil is described. Finally, the AC losses under different conditions are presented and analyzed.

\section{WORKING PRINCIPLES OF THE SCSFCL}

As shown in Fig. 1, in SCSFCL, there is one superconducting DC coil, two copper AC coils, and two iron cores. The two AC coils are connected in series with the power system, and the directions of the magnetic field generated by each oppose each other. The DC coil is connected to an independent DC power source. 


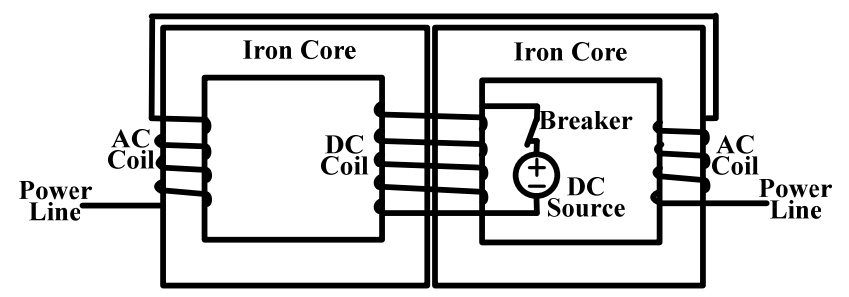

Fig. 1. Schematic diagram of the main components of an SCSFCL.

Under normal power system conditions, the SCSFCL works in its normal non-limiting state, which is also the state it operates in for most of the time. In this state, rated current will flow through the AC coils, and a large DC magnetic field bias is generated in the iron cores by the large current in the superconducting DC coil. The iron cores are kept in deep saturation with low permeability during each AC cycle. As the inductance is proportional to permeability, the reactance of the SCSFCL is small, and it has a minimal effect on the rest of the power system.

When short-circuit faults occur, a large fault current will flow through its AC coils, and the SCSFCL will enter its current-limiting state. For the active type SCSFCL, the DC current will be cut off immediately, and for the passive type SCSFCL, the same DC bias will be maintained, regardless of the short-circuit fault. For both these two types, due to the large fault current, the iron cores will no longer always be in deep saturation. When the iron cores then work in the linear region of the $B-H$ curve, the permeability increases by thousand times, so the average reactance of the SCSFCL is large. This reactance enables the SCSFCL to limit the fault current.

\section{Simulation METHOD FOR MODELING AC LOSSES IN THE SCSFCL'S SUPERCONDUCTING DC COIL}

In consideration of the main operating features of the SCSFCL, the simulation method is presented in this section. Firstly, the two non-linear problems are decoupled. The strategy, as well as the corresponding difficulties, to simulate the $3 \mathrm{D}$ model is given. Finally, a feasible, simplified 2D simulation method to estimate the worst-case AC losses is presented.

\section{A. Decoupling the two non-linear problems}

There are two non-linear materials in the SCSFCL: one is the soft iron core with non-linear magnetic properties, which is determined by the material's $B-H$ curve, and the other is the superconducting materials, which have non-linear electrical properties that can be described by an $E-J$ power law. To simulate the characteristics of the superconducting DC coil in the SCSFCL, these two coupled non-linear problems must be solved simultaneously. However, one challenge is the huge geometric scale difference between these two parts. The thickness of the superconducting layer in a coated conductor is $1 \mu \mathrm{m}$, and the height of the iron core in the SCSFCL under research is $4.275 \mathrm{~m}$, as shown in Fig. 2. The latter is more than four million times larger in scale than the former.
Additionally, the number of turns/layers in the superconducting DC coil in the model under research is up to 7800 in this particular design, making the simulation of just the superconducting coils in 3D difficult. It is difficult to even simulate a copper DC coil with wire of such a large aspect ratio and so many turns, and far more difficult to calculate these two non-linear materials in the SCSFCL at the same time, so these need to be decoupled first.

For a solenoid model in quasi-steady-state, in the domain where the distance from the coil is much larger than the wire dimensions, the magnetic field when all the wires are modeled in detail and when the wires are simplified as one single turn is almost the same without much error. The normal nonlimiting state of the SCSFCL is quasi-steady-state. Hence, as the first step in our method, the superconducting DC coil is simplified as one single-turn homogenized coil. Although the current density is taken as uniformly distributed and the spaces between the superconducting tapes are ignored, the magnetic field in the iron cores and air sub-domain not close to the superconducting coils are accurate enough, according to Ampere's law.

As Fig. 2 shows, the DC superconducting coil is enclosed with an appropriately small boundary, so as not to include any iron core, and the magnetic field on the boundaries is calculated. Next, with this information, we can remove all the parts outside the small boundary, and consider the influence the iron cores has on the parts inside the small boundary by applying Dirichlet boundary conditions with the magnetic field distribution obtained. Therefore, we can focus on the superconducting DC coil alone, and the two non-linear problems are decoupled.

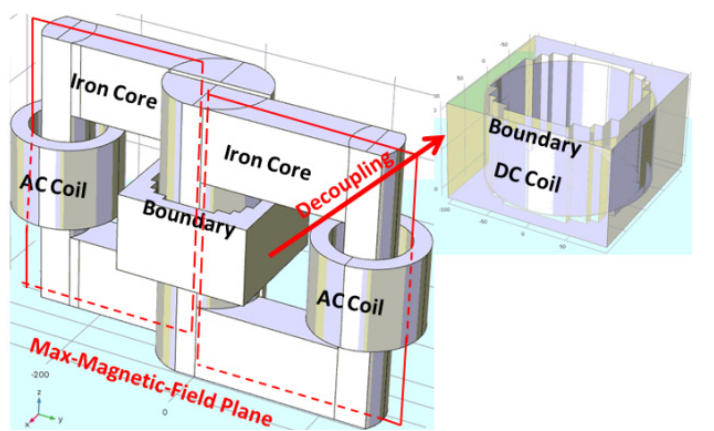

Fig. 2. Decoupling the two non-linear problems: soft iron core with non-linear magnetic properties, and superconducting materials with non-linear electrical properties.

\section{B. Strategy \& difficulties in obtaining AC losses with a $3 D$ model}

After the preceding decoupling is carried out, the superconducting DC coil can be analyzed separately. However, as there are 120 coil layers (60 double pancakes of 130 individual turns), and up to 7800 turns, it is still difficult to simulate all of the coils of so many turns directly at one time.

Inspired by the simulation method presented in [9], we divide the decoupled domain into 120 sub-domains, as Fig. 3 shows, so that the AC losses in each layer could be calculated in parallel by importing boundary conditions and employing 
3D equivalent anisotropic homogeneous bulk model [10]. First, we need to more accurately determine the boundary conditions for the boundaries between the 120 layers. Hence, the one single-turn homogenized DC coil inside the small boundary is re-built with individual, homogenized layers considering the real tape height. The magnetic field on the boundaries between the 120 layers is then calculated, and then each of these layers can be simulated separately.

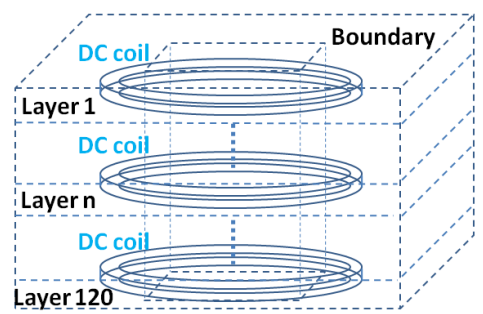

Fig. 3. Strategy to decouple the individual coil layers in the entire superconducting DC coil to simulate the AC losses of the entire coil.

Although this strategy seems theoretically feasible, when this was attempted in $3 \mathrm{D}$, there is a large scale difference between the superconducting tape height and the circumference of the coil. The latter is more than 1400 times of the former, and additionally the mapped mesh size in the tapes is much smaller than the tape height, which makes the mesh in each layer either too small to converge quickly or too large to converge. Thus, in order to calculate the characteristics of the superconducting coils, the model needs further simplification, which is described in the next section.

\section{Further simplification to a $2 \mathrm{D}$ model}

The problem is not axisymmetric due to the varying electromagnetic boundary conditions, and as such the coil cannot be simplified directly. In order to analyze the coil in $2 \mathrm{D}$, we find the region of highest magnetic field for the radial cross sections of the coil layer, and estimate the AC loss for a conservative design. Although this method is an overestimation based on the worst-case scenario, it can still provide crucial AC loss information for our design and can also indicate trends in the superconducting coil's properties/performance when some design parameters are changed.

Generally, the larger the magnitude of the magnetic field and its variation are, the larger the AC loss is, and the smaller the critical current is. The leakage magnetic field close to the inner core is much larger than that in other locations. As shown in Figs. 2 and 4, for one iron core, the direction of the pillars and yoke is parallel to the $y-z$ plane, so due to the direction of the iron core, the $x$ component of the magnetic field is small enough to be neglected compared to the $y$ and $z$ components. Hence, the coil cross-sections cut by the $y-z$ planes can be treated as 2D infinitely long model. Among these $y-z$ planes inside the core, the largest leakage magnetic field occurs in the middle one, named as the "Max-MagneticField" plane, which contains the "Max Surface Section." The radial coil cross-section in the worst-case scenario is then named the "Max Coil Section", which is nearest to the "Max
Surface Section." For the conservative design, where the AC loss is maximized, we can apply the $2 \mathrm{D}$ boundary conditions of the "Max-Surface Section" to the "Max Coil Section," and consider the dependence of the critical current on the magnetic field magnitude and the corresponding angle to the normal direction (radial direction in this model) of the superconducting tape surface $[11,12]$.

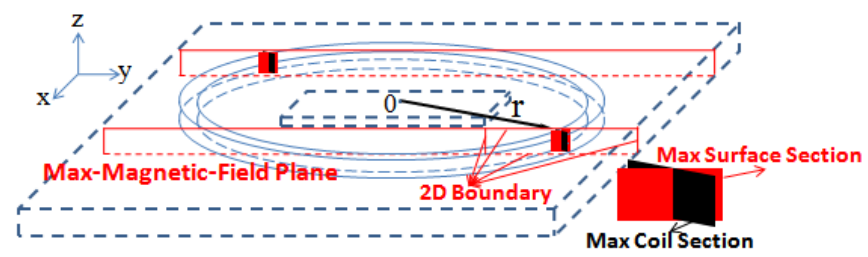

Fig. 4. Single coil layer model example of the $2 \mathrm{D}$ simplified method to calculate worst-case scenario AC losses for a conservative design.

To calculate the 2D model for the "Max Coil Section" geometry with the "Max Surface Section" boundary conditions, the boundary conditions from the decoupled 3D model with the individual, homogenized layers are applied to the 2D model, as shown in Fig. 5. We further simplify the 2D model by employing the homogenized model presented in [13, 14]. Firstly, the thickness of the superconducting tape in each turn is artificially expanded as the average thickness for each turn, thus forming a bulk, homogenized approximation. At the same time, the critical current for each tape is reduced by the same multiple [13, 14]. As all the turns in one bulk element have the same average current density, they can be re-meshed with elements of arbitrary width, forming several "turns" with the similar distributions of current densities.

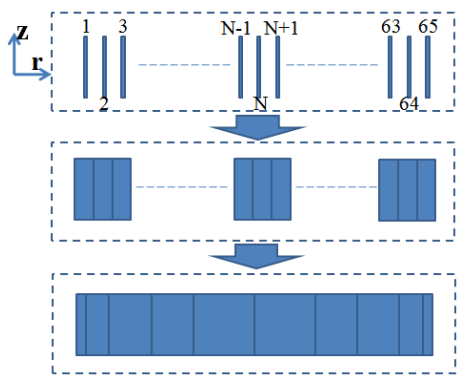

Fig. 5. Implementation of the simplified 2D homogenized model to simulate the superconducting coils.

As shown in $[11,14]$ the governing equations $(\boldsymbol{H}$ formulation) for modeling the superconducting DC coil are as follows.

$$
\begin{gathered}
\nabla \times \mathbf{E}+d \mathbf{B} / d t=0 \\
\nabla \times \mathbf{H}=\mathbf{J} \\
\mathbf{B}=\mu_{0} \mu_{r} \mathbf{H} \\
\mathbf{E}=\frac{E_{0}|\mathbf{J}|^{n-1}}{J_{c}^{n}(B, \theta)} \mathbf{J}
\end{gathered}
$$

where (1) is Faraday's law, (2) is Ampere's law, (3) is the $B-H$ relationship, and (4) is the $E-J$ power law relationship, where $E_{\mathrm{c}}=10^{-4} \mathrm{~V} / \mathrm{m}, n=21[12] . J_{c}(B, \theta)$ at $77 \mathrm{~K}$ can be obtained 
from the critical current, $I_{c}(B, \theta)$, of the superconductor, divided by the cross-sectional area:

$$
\begin{aligned}
& I_{c}(B, \theta)=\frac{K_{0}}{\left(\frac{B}{\beta_{0}}+1\right)^{a_{0}}}+\frac{K_{1}}{\left(\frac{B}{\beta_{1}}+1\right)^{a_{1}}} . \\
& {\left[\left(\frac{B}{\beta_{\omega}}+1\right)^{\frac{6}{5}} \cos ^{2}\left(\theta-\theta_{0}\right)+\sin ^{2}\left(\theta-\theta_{0}\right)\right]^{-\frac{1}{2}}}
\end{aligned}
$$

where $K_{0}=14.625 \mathrm{~A}, K_{1}=110.65 \mathrm{~A}, \beta_{0}=13.8 \mathrm{~T}, \beta_{1}=13.8 \mathrm{~T}$, $\beta_{\omega}=0.2792 \mathrm{~T}, a_{0}=1.3, a_{1}=0.809$ and $\theta_{0}=-0.180^{\circ}$, which are assumed from the fitting presented in [12].

As shown in Fig. 6, the AC loss, $Q_{\text {ave }}$, is calculated by:

$$
Q_{\text {ave }}=\frac{\int_{0.5 T}^{1.5 T}\left(\int_{S} \boldsymbol{E} \cdot \boldsymbol{J} d s\right) d t}{T}
$$

where the $T$ is the period of each AC cycle, $d s$ is the crosssectional area.

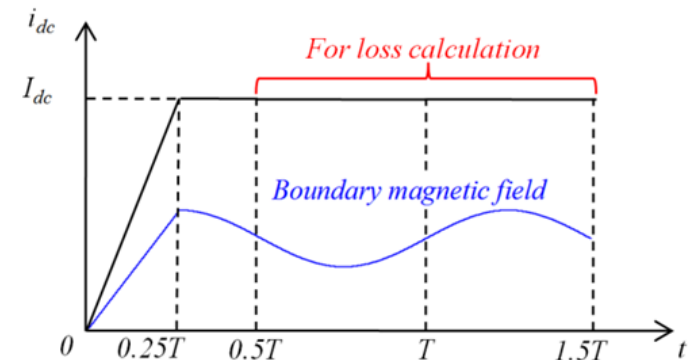

Fig. 6. Time dependence of the applied DC current and applied magnetic field boundary conditions in the model.

\section{SIMULATION RESULTS AND ANALYSIS}

The results for a $500 \mathrm{kV}$ SCSFCL (key parameters listed in Table 1, model shown in Fig. 2) using our simulation method is presented in Fig. 7.

TABLE I

KEY PARAMETER OF THE $500 \mathrm{KV}$ SCSFCL

\begin{tabular}{cc}
\hline Item & Quantity \\
\hline \hline Iron core diameter (DC coil) & $1560 \mathrm{~mm}$ \\
Iron core diameter (AC coils) & $860 \mathrm{~mm}$ \\
Core height (above core window) & $1145 \mathrm{~mm}$ \\
Core (window) depth & $714 \mathrm{~mm}$ \\
Core (window) height & $1985 \mathrm{~mm}$ \\
Core (window) width & $1465 \mathrm{~mm}$ \\
DC coil diameter (inner, outer) & $1908 \mathrm{~mm}, 1939.2 \mathrm{~mm}$ \\
AC coil diameter (inner, outer) & $1150 \mathrm{~mm}, 1660 \mathrm{~mm}$ \\
AC coil height & $1285 \mathrm{~mm}$ \\
AC excitation & $117.6 \mathrm{kA} \cdot$ turns \\
DC excitation & $60 * 7800$ A·turns \\
Superconducting tape width & $4.3 \mathrm{~mm}$ \\
Double pancake height & $15.5 \mathrm{~mm}$ \\
Iron core material & B30P105 by BAOSTEEL \\
\hline
\end{tabular}

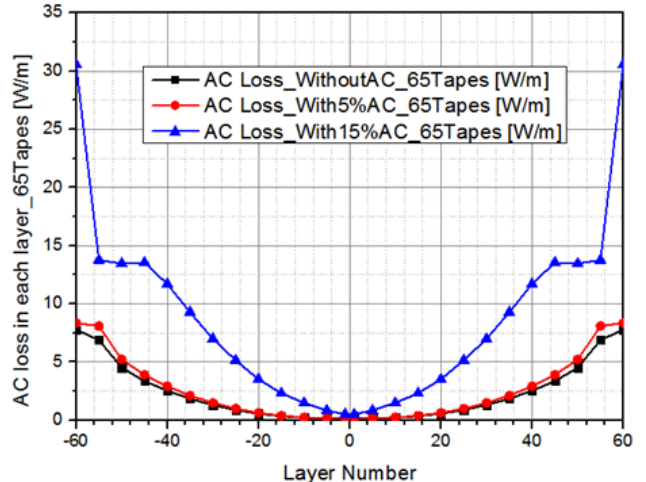

Fig. 7. Calculated AC losses in each coil layer of the superconducting DC coil, with and without an AC component in the DC current.

From the results, it can be found that from the central coil layer (layer 0 ) to the outermost coil layer (layer 60 or -60 ), the $\mathrm{AC}$ loss increases. Moreover, the variation of the AC loss in the central few layers and outermost few layers is small, and in the layers between them, it is an almost quadratic curve. The reason for these two phenomena is that the magnetic field in the central few layers is almost parallel to the tape surface. As the layer approaches the outermost one, the magnetic field component normal to the tape surface increases gradually, and near the outermost few layers, the normal component tends towards its maximum. It is indicated here that the outermost layers are the layers most likely to quench in the whole coil, and measures like shielding the magnetic field should be taken to protect these few layers. To calculate the AC loss of the whole coil, there is no need to calculate all of the individual layers one by one. We need only to calculate the outermost few layers carefully and pick several central and middle layers, and the losses in other layers can be estimated by interpolation.

It is also found that a 5\% AC component of the overall DC current does not influence the AC loss significantly. However, with a $15 \%$ AC component, the AC loss increases significantly, and the outermost layer has quenched in this 2D model, and is likely to have quenched in 3D model, so measures should be taken to avoid this problem.

\section{CONCLUSIONS}

1. A simplified $2 \mathrm{D}$ method is presented in this paper to estimate the AC losses of the superconducting DC coil in the SCSFCL in the normal, non-limiting state, which is its usual operating state. Although this method is an overestimation based on the worst-case scenario, it can still provide crucial $\mathrm{AC}$ loss information for the design and can also indicate trends in the superconducting coil's properties/performance when some design parameters are changed.

2. Employing the simulation method we presented, the properties of the superconducting coils in the SCSFCL are calculated for the first time. It is found that the outermost layers have much larger AC loss than the central layers. Hence, the outermost coil layer is the layer most likely to quench, which should be protected by measures like magnetic shielding. It is also found the AC loss can be estimated by picking several key layers in the central, middle and outermost 
layers and then interpolating, instead of calculating all of the individual layers one by one.

3. For an increase in the AC component in the DC current in the coil, the AC loss increases sharply when AC component is over $5 \%$, so measures should be taken to avoid this problem.

4. The next logical step is to extend this model strategy to a $3 \mathrm{D}$ coil model, but this is a difficult challenge due to the large scale difference between the superconducting tapes and the circumference of the superconducting coil layer. However, this will improve the accuracy of the calculation and will enable more detailed design and analysis of the SCSCFL.

\section{REFERENCES}

[1] Y. Nikulshin et al., "Saturated Core Fault Current Limiters in a Live Grid," IEEE Trans. Appl. Supercond., vol. 26, no. 3, Apr. 2016, Art. ID 5601504.

[2] Y. Jia et al., "Cognition on the Current-Limiting Effect of SaturatedCore Superconducting Fault Current Limiter," IEEE Trans. Magn., vol. 51, no. 11, Nov. 2015, Art. ID 8003004.

[3] J. B. Cui et al., "Safety Considerations in the Design, Fabrication, Testing, and Operation of the DC Bias Coil of a Saturated Iron-Core Superconducting Fault Current Limiter," IEEE Trans. Appl. Supercond., vol. 23, no. 3, Jun. 2013, Art. ID 5600704.

[4] H. Heydari and R. Sharifi, "Three-Dimensional Pareto-Optimal Design of Inductive Superconducting Fault Current Limiters," IEEE Trans. Appl. Supercond., vol. 20, no. 5, pp. 2301-2311, Oct. 2010.

[5] Y. Jia et al., "Simulation Method for Current-Limiting Effect of Saturated-Core Superconducting Fault Current Limiter," IEEE Trans. Appl. Supercond., vol. 26, no. 4, Jun. 2016, Art. ID 5601404.

[6] S-H. Lim, Y-P. Kim, and S-C. Ko, "Effect of Peak Current Limiting in Series-Connection SFCL With Two Magnetically Coupled Circuits Using E-I Core," IEEE Trans. Appl. Supercond., vol. 26, no. 3, Apr. 2016, Art. ID 5600404.

[7] S. M. Gunawardana, P. A. Commins, J. W. Moscrop, and S. Perera, "Transient Modelling of Saturated Core Fault Current Limiters," IEEE Trans. Power Del., to be published [DOI: 10.1109/TPWRD.2016.2524647].

[8] J. C. Knott and J. W. Moscrop, "Increasing Energy Efficiency of Saturated-Core Fault Current Limiters With Permanent Magnets," IEEE Trans. Magn., vol. 49, no. 7, pp. 4132-4136, Jul. 2013.

[9] L. Quéval, V. M. R. Zermeno, and F. Grilli, "Numerical models for ac loss calculation in large-scale applications of HTS coated conductors," Supercond. Sci. Technol., vol. 29, no. 2, Feb. 2016, Art. ID 024007.

[10] V. M. R. Zermeño and F. Grilli, "3D modeling and simulation of $2 \mathrm{G}$ HTS stacks and coils," Supercond. Sci. Technol., vol. 27, no. 4, Apr. 2014, Art. ID 044025.

[11] D. Hu et al., "Modeling and Comparison of In-Field Critical Current Density Anisotropy in High-Temperature Superconducting (HTS) Coated Conductors," IEEE Trans. Appl. Supercond., vol. 26, no. 3, Apr. 2016, Art. ID 6600906.

[12] E. Pardo, "Modeling of screening currents in coated conductor magnets containing up to 40000 turns," Supercond. Sci. Technol., vol. 29, no. 8, Aug. 2016, Art. ID 085004.

[13] V. M. R. Zermeno et al., "Calculation of alternating current losses in stacks and coils made of second generation high temperature superconducting tapes for large scale applications," J. Appl. Phys., vol. 114, Nov. 2013, Art. ID 173901.

[14] J. Xia et al., "Electromagnetic modeling of REBCO high field coils by the $H$-formulation," Supercond. Sci. Technol., vol. 28, no. 12, Dec. 2015, Art. ID 125004. 